КЛИНИЧЕСКИЕ РЕКОМЕНДАЦИИ ПО ВРОЖДЕННОМУ

ГИПОТИРЕОЗУ ЕВРОПЕЙСКОГО ОБЩЕСТВА ДЕТСКИХ ЭНДОКРИНОЛОГОВ

(ЕSРЕ) И ЕВРОПЕЙСКОГО ЭНДОКРИНОЛОГИЧЕСКОГО ОБЩЕСТВА (ЕSО):

ОСНОВНЫЕ ПОЛОЖЕНИЯ И КОММЕНТАРИИ

\author{
( Е.В. Шрёдер*, Т.Ю. Ширяева, Е.В. Нагаева, О.Б. Безлепкина
}

ФГБУ «Национальный медицинский исследовательский центр эндокринологии» Минздрава России, Москва, Россия

Врожденный гипотиреоз - актуальная клиническая проблема в педиатрической практике. Предыдущие Европейские клинические рекомендации были опубликованы в 2014 г. Настоящие клинические рекомендации основаны на данных, опубликованных с января 2013 по начало 2020 гг. Основные разделы рекомендаций: результаты неонатального скрининга, диагностика и сроки начала лечения, мониторинг, генетика врожденного гипотиреоза, генетическое консультирование семей.

КЛЮЧЕВЫЕ СЛОВА: врожденный гипотиреоз; неонатальный скрининг; тиреотропный гормон (ТТГ); тироксин (T 4 ); щитовидная железа; генетика врожденного гипотиреоза.

\title{
CONSENSUS GUIDELINES OF CONGENITAL HYPOTHYROIDISM BY THE EUROPEAN SOCIETY FOR PEDIATRIC ENDOCRINOLOGY AND THE EUROPEAN SOCIETY FOR ENDOCRINOLOGY: KEY POINTS AND COMMENTS
}

\author{
(c) Ekaterina V. Shreder*, Tatiana Y. Shiryaeva, Elena V. Nagaeva, Olga B. Bezlepkina
}

Endocrinology Research Centre, Moscow, Russia

Congenital hypothyroidism is an actual clinical problem in pediatric practice. Previous clinical guidelines were published in 2014. The presented clinical recommendations are based on articles published from January 2013 to early 2020. The consensus guidelines include such sections as results of neonatal screening, diagnostics and criteria for treatment, lifelong follow-up, genetics of congenital hypothyroidism, family genetic counselling. The key points of new congenital hypothyroidism guidelines were commented by pediatric endocrinologists of Russians.

KEYWORDS: congenital hypothyroidism; neonatal screening; thyroid-stimulating hormone (TSH); thyroxine (T4); thyroid glands; genetic of congenital hypothyroidism.

\section{ВВЕДЕНИЕ}

Врожденный гипотиреоз (ВГ) представляет собой гетерогенную группу заболеваний, обусловленных морфофункциональной незрелостью щитовидной железы (ЩЖ), гипоталамо-гипофизарной системы, а также нарушенным действием гормонов ЩЖ (дисгормоногенезом), манифестирует при рождении и в результате приводит к дефициту гормонов щЖ различной степени тяжести.

Гормоны ЩЖ играют решающую роль в развитии нервной системы, поэтому своевременная диагностика и лечение ВГ позволяют предотвратить грубые неврологические и психические нарушения. Ранняя диагностика заболевания возможна только при проведении неонатального скрининга. Впервые скрининг на ВГ был осуществлен в 1974 г. в Квебеке (Канада) [1], в настоящее время проводится в большинстве развитых стран мира. В Российской Федерации скрининг на ВГ в пилотных регионах страны был начат в 1993 г., в настоящее время является обязательной процедурой обследования новорожденных.

В 2014 г. в журнале Hormone Research in Paediatrics был опубликован международный консенсус по скринингу, диагностике и лечению ВГ, обобщающий многолетние дан- ные, в том числе по проведению скрининга на ВГ [2]. Обновленные клинические рекомендации по ВГ представлены в декабрьском номере журнале Thyroid в 2020 г. [3]. В 2014 г. опубликованы отечественные клинические рекомендации по диагностике и лечению ВГ [4], в настоящее время профессиональным сообществом обсуждается актуализированный вариант данных рекомендаций [5]. Предлагаемая статья является переводом основных положений Европейских рекомендаций 2020 г. с комментариями.

Сила (уровень) рекомендаций и их качество ранжированы в соответствии с системой GRADE (the Grading of Recommendations, Assessment, Development and Evaluation) [6].

\section{1. НЕОНАТАЛЬНЫЙ СКРИНИНГ}

1.1. Преимущества скрининга на ВГ.

- Раннее выявление ВГ с помощью неонатального скрининга и своевременно начатое лечение предотвращают необратимую задержку развития нервной системы и улучшают прогноз $(1 / \bigoplus \bigoplus \bigoplus)$.

- Скрининг на ВГ должен быть внедрен во всем мире $(1 / \bigoplus \oplus \oplus)$. 
1.2. Методология и эффективность скрининга на ВГ.

- Частота первичного ВГ составляет 1 случай на 2000-3000 и частично зависит от стратегии скрининга (применяемого метода исследования); заболеваемость центральным ВГ составляет около 1 случая на $16000(1 / \oplus \oplus \oplus)$.

- Главная цель неонатального скрининга на ВГ - выявление всех форм первичного ВГ - легкой, средней тяжести и тяжелой; наиболее чувствительным исследованием для выявления первичного ВГ является определение уровня тиреотропного гормона (ТТГ) $(1 / \oplus \oplus \oplus)$.

- При наличии финансовых ресурсов для скрининга центрального ВГ рекомендуется к ТТГ добавить измерение общего $\left(\mathrm{T}_{4}\right)$ или свободного тироксина (св. $\left.\mathrm{T}_{4}\right)$ $(2 / \oplus \oplus \circ)$.

Комментарии. В Европейском консенсусе 2014 г. [2] приоритетным направлением было выявление всех форм первичного $\mathrm{B}$, обновленные рекомендации освещают вопрос скрининга центрального ВГ. Распространенность первичного ВГ в РФ по результатам неонатального скрининга составляет 1 на 3617 новорожденных [7]. В настоящее время скрининг на центральный ВГ в РФ не проводится [4].

\section{3. Тактика после проведения скрининга для новорожденных, подверженных высокому риску} развития $\mathrm{B} Г$ 。

- Некоторые группы детей (недоношенные новорожденные, новорожденные с низкой массой тела при рождении, дети с сопутствующими заболеваниями в неонатальном периоде) могут иметь ложноотрицательный результат неонатального скрининга или высокий риск легкой формы ВГ, не выявленного при неонатальном скрининге; для этих групп предполагается повторное обследование в возрасте от 10 до 14 дней $(1 / \bigoplus \circ \circ)$.

- У пациентов с синдромом Дауна рекомендуется определять уровень ТТГ к концу первого месяца жизни $(1 / \oplus \oplus \circ)$.

- Первичный скрининг у пробанда может быть ложноотрицательным; необходимо рассмотреть вопрос о проведении повторного скрининга у однополых близнецов. У сиблинга, не имеющего ВГ, уровень ТТГ должен контролироваться в более позднем возрасте настолько долго, насколько это возможно (2/Фо०).

- При клиническом подозрении на гипотиреоз, несмотря на нормальный уровень ТТГ по скринингу, необходимо дальнейшее обследование (редко, но встречаются ложноотрицательные результаты), особенно у детей с отягощенным наследственным анамнезом по центральному ВГ (2/Фо०).

Комментарии. Ложноотрицательный результат неонатального скрининга у перечисленных выше категорий новорожденных может быть обусловлен незрелостью гипоталамо-гипофизарно-тиреоидной оси $[8,9]$. Также следует иметь в виду высокую частоту транзиторных форм ВГ у данных пациентов, что требует повторного обследования для уточнения диагноза в более позднем возрасте.

Как в зарубежных рекомендациях 2014 г. [2], так и в Российских [4] не освещены сроки контроля уровня ТТГ для детей с синдромом Дауна. Поскольку большин- ство новорожденных с этим синдромом при рождении имеют тяжелую сопутствующую патологию, синтез ТТГ может быть нарушен, что приводит к ложноотрицательному результату неонатального скрининга и требует повторного определения уровня ТТГ в возрасте 3-4 нед [10, 11].

Особая ситуация складывается при многоплодной беременности. Из-за смешивания крови плодов концентрация ТТГ у близнеца с ВГ может быть ниже истинных значений, и ВГ может не выявляться при скрининге, в связи с чем необходим повторный контроль у однополых близнецов $[12,13,14]$.

\section{2. ДИАГНОСТИКА И КРИТЕРИИ ДЛЯ НАЧАЛА ЛЕЧЕНИЯ}

\section{1.Биохимические критерии, используемые при} принятии решения о начале лечения ВГ.

- При отклонении результатов неонатального скрининга от референсных значений, новорожденный должен быть направлен в специализированный центр $(1 / \oplus \oplus \circ)$.

- При положительном результате скрининга необходимо провести подтверждающее исследование, включающее определение уровня св. Т 4 и ТТГ венозной крови $(1 / \oplus \oplus \circ)$.

- Если уровень св. Т в сыворотке крови ниже порогового значения, а ТТГ выше референсного интервала, показано немедленное назначение лечения левотироксином $(1 / \oplus \oplus \oplus)$.

- Если уровень ТТГ в сыворотке крови составляет более $20 \mathrm{ME} / л$ при подтверждающем исследовании (приблизительно на 2-й неделе жизни), лечение следует начинать, даже если св. $T_{4}$ в норме $(2 / \bigoplus \circ \circ)$.

- Если уровень ТТГ в сыворотке крови составляет 6-20 MЕ/л после 21-дневного возраста у здорового новорожденного с уровнем св. $\mathrm{T}_{4}$ в пределах возрастного референсного интервала, предлагается: немедленно начать лечение левотироксином и провести повторное исследование на более позднем этапе (на фоне отмены терапии) либо воздержаться от лечения, но повторить обследование через

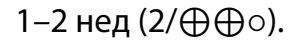

- Если уровень св. Т ${ }_{4}$ низкий, уровень ТТГ низкий, нормальный или немного повышенный, диагностика центрального ВГ должна быть продолжена $(1 / \bigoplus \bigoplus$ о).

- У новорожденных с центральным ВГ рекомендуется начинать лечение левотироксином только после исключения надпочечниковой недостаточности; если центральная надпочечниковая недостаточность не может быть исключена, лечение глюкокортикоидами должно предшествовать лечению левотироксином, чтобы предотвратить возможное развитие криза надпочечниковой недостаточности (2/Фо०).

- В странах или регионах, где гормональные исследования для оценки функции ЩЖ недоступны, лечение левотироксином следует начинать, если концентрация ТТГ в пятне фильтровальной бумаги составляет более $40 \mathrm{ME} / л$ (2/Ф००).

Комментарии. Раннее выявление и своевременно начатое лечение ВГ (в течение первых 2 нед жизни) имеют важное значение для нейрокогнитивного развития и роста ребенка. 
В РФ рекомендуется забор образцов капиллярной крови для проведения неонатального скрининга у доношенных новорожденных на 3-4-е сутки жизни, у недоношенных - не ранее 7 сут $[4,15]$. Референсный уровень ТТГ зависит от диагностических наборов, например, для иммунодиагностической системы «Делфия» («Делфия неонатал ТТГ») нормальным является уровень ТТГ капиллярной крови менее 9 мЕд/л.

В случае повышенного уровня ТТГ проводится повторное определение ТТГ из того же образца крови, и при повторно повышенном уровне неонатального ТТГ срочно уведомляются врачи детской поликлиники, где ребенку производится забор венозной крови для определения уровней ТТГ и св. Т. Если уровень неонатального ТТГ был выше $40 \mathrm{mEд/л} \mathrm{-} \mathrm{не} \mathrm{дожидаясь} \mathrm{результатов}$ из венозной крови, назначается заместительная терапия левотироксином. Во всех остальных случаях тактика определяется после получения результатов ТТГ и св. Т в сыворотке.

При получении результатов в пределах референсных значений терапия отменяется. Остальные рекомендации аналогичны рекомендациям Европейского консенсуса 2020 г. [3].

Остается спорным вопрос о пользе назначения лечения левотироксином новорожденных с «умеренным» гипотиреозом (уровень ТТГ 6-20 мЕ/л и нормальный св. Т 4 ) $[16,17,18]$. Динамическое наблюдение за детьми с ВГ осуществляется детским эндокринологом.

\section{2. Оповещение о положительном результате неонатального скрининга.}

- Положительный результат неонатального скрининга должен быть сообщен специалистом (детским эндокринологом, педиатром или врачом общей практики) по телефону либо лично и дополнен письменной информацией для семьи ребенка (2/๑оо).

Комментарии. В Российской Федерации информация о повышенном уровне ТТГ и необходимости дополнительного обследования (определение ТТГ и св. Т в сыворотке крови) передается сотрудниками медикогенетических лабораторий в детскую поликлинику.

\section{3. Методы визуализации при ВГ.}

- Пациентам с установленным диагнозом ВГ настоятельно рекомендуется начать лечение левотироксином до инструментальной визуализации щЖ $(1 / \bigoplus \bigoplus$ $)$.

- Рекомендуется визуализировать щЖ при помощи радиоизотопного сканирования (сцинтиграфия) или ультразвукового исследования (УЗИ) либо с исполь-

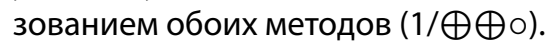

- Для оценки тяжести внутриутробного гипотиреоза может быть выполнена рентгенография коленного сустава $(2 / \bigoplus о \circ)$.

Комментарии. Несмотря на то что этиология не влияет на тактику лечения, всем детям с ВГ рекомендовано проведение визуализации тиреоидной ткани. В настоящее время в РФ детям с ВГ с целью топической диагностики рутинно проводится только УЗИ ЩЖ, тиреосцинтиграфия не получила широкого применения в связи с низкой доступностью и выполняется в редких случаях, как правило, детям старшего возраста.
Визуализация с использованием УЗИ и сцинтиграфии обеспечивает высокое анатомическое и функциональное разрешение, позволяющее различить перманентный и транзиторный ВГ [19]. Сочетание двух методов особенно эффективно в случаях аплазии и эктопии ЩЖ [20] и устраняет необходимость дальнейшего диагностического поиска.

\section{4. Сопутствующие пороки развития и синдромы.}

- Bсе новорожденные с высокой концентрацией ТТГ должны быть тщательно обследованы на наличие дисморфических особенностей, свидетельствующих о синдромальной форме ВГ, а также о врожденных пороках развития (в особенности пороков сердца) $(1 / \bigoplus \bigoplus \bigoplus)$.

\section{3. ЛЕЧЕНИЕ И МОНИТОРИНГ ВГ}

\section{1. Начало лечения первичного ВГ.}

- Для лечения ВГ единственный рекомендованный

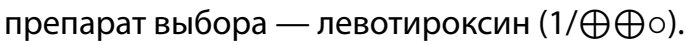

- Лечение левотироксином следует начинать как можно скорее, не позднее чем через 2 нед после рождения ребенка или сразу после подтверждения диагноза (на основании гормонов в сыворотке крови) $(1 / \oplus \bigoplus$ $)$.

- Начальная максимальная доза левотироксина должна составлять не более 15 мкг/кг/сут, с учетом различ-

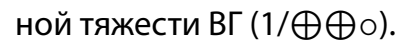

- В случае тяжелых форм ВГ: низкие уровни общего $\mathrm{T}_{4}$ и/или св. $\mathrm{T}_{4}$ (в сыворотке крови до лечения менее 5 пмоль/л) в сочетании с повышенным уровнем ТТГ (выше нормального диапазона с учетом гестационного возраста и возраста с момента рождения), следует начинать лечение с максимальной начальной дозы

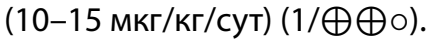

- Младенцам с умеренным ВГ (до лечения св. Т4 в сыворотке более 10 пмоль/л в сочетании с повышенным ТТГ) лечение следует начинать с начальной дозы 10 мкг/кг в сутки; при повышенном ТТГ и референсном св. $\mathrm{T}_{4}-$ с начальной дозы

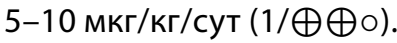

- Левотироксин следует принимать перорально, 1 раз в день $(1 / \bigoplus \bigoplus$ $)$.

- Доказательства в пользу использования оригинального препарата левотироксина против дженерика неоднозначны, но, основываясь на личном опыте/ экспертном мнении, рекомендуется оригинальный

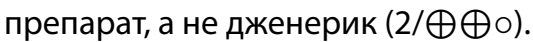

Комментарии. На сегодняшний день отсутствуют рандомизированные клинические исследования, в которых был бы указан конкретный подход к лечению, основанный на высококачественных доказательствах. Первоначально изучалось соматическое развитие с оценкой роста и полового созревания, но в дальнейшем фокусом исследований стала оценка когнитивного статуса (IQ). Самый высокий уровень доказательств был получен в тех исследованиях, которые оценивали IQ у пациентов с ВГ, где контрольной группой служили здоровые сибсы. Исследования указывают на выраженное влияние двух основных факторов, определяющих когнитивный исход, возраста начала лечения левотироксином и стартовой 
дозы препарата. Основываясь на литературных данных, можно сделать вывод, что ребенок даже с тяжелой формой ВГ может достичь нормального IQ, который не отличается от здоровых братьев и сестер, если лечение левотироксином начинается до достижения возраста 10 дней и начальная доза составляет не менее 10 мкг/кг/сут, а при тяжелых формах - 15 мкг/кг/сут [21, 22].

\section{2. Мониторинг лечения при первичном ВГ.}

- Рекомендуется измерять концентрацию св. Т и ТТГ в сыворотке крови до или по крайней мере через 4 ч после приема левотироксина $(1 / \bigoplus \bigoplus$ $)$.

- Рекомендуется оценивать св. Т и ТТГ в соответствии с возрастными референсными интервалами $(1 / \bigoplus \bigoplus$ $)$.

- Основная цель лечения новорожденных с первичным ВГ заключается в быстром повышении уровня св. $\mathrm{T}_{4}$ что приводит к нормализации уровня ТТГ в сыворотке крови; в дальнейшем ТТГ следует поддерживать в пределах референсного интервала.

- Если ТТГ находится в референсном интервале, то допустима концентрация св. Т 4 выше верхнего предела референсного интервала и рекомендовано поддер-

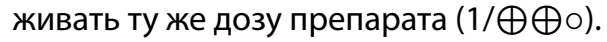

- Любое снижение дозы левотироксина не должно основываться на единичном превышении нормальной концентрации св. Т $4^{\prime}$ если только ТТГ не подавлен (т. е. ниже нижней границы референсного интервала) или нет признаков тиреотоксикоза (например, тремор

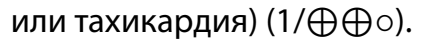

- Первая клиническая и биохимическая оценка должна проводиться через 1-2 нед после начала лечения левотироксином (не позднее 1 нед в случае начальной дозы 50 мкг в день и более) (1/Фо०).

- Последующий контроль должен проводиться каждые 2 нед до нормализации уровня ТТГ в сыворотке крови, после чего частота может быть уменьшена до 1 раза в 1-3 мес до достижения 12-месячного возраста (1/Фо०).

- В возрасте от 12 мес до 3 лет периодичность контроля может быть уменьшена до 1 раза в 2-4 месяца; после этого оценка должна проводиться каждые 3-6 мес до завершения роста (1/Фоо).

- Если обнаружены отклонения св. Т или ТТГ от референсных значений или результат сомнительный, то частота обследований должна быть увеличена (2/๑о०).

- После изменения дозы или замены препарата следует провести дополнительный контроль через 4-6 нед (2/Фо०).

- В отличие от взрослых, у новорожденных, младенцев и детей левотироксин можно принимать вместе с пищей (без соевого белка и растительных волокон); препарат следует принимать ежедневно в одно и то же время с целью обеспечения лучшей абсорбции препарата и при этом лучшего титрования дозы (2/๑о०).

- В случае неожиданной необходимости увеличения дозы, снижения абсорбции или увеличения метаболизма тироксина при других заболеваниях (например, желудочно-кишечного тракта) следует рассмотреть вопрос о раздельном приеме пищи или медикаментов (2/Фоо); наиболее частой причиной может быть несоблюдение рекомендаций, особенно у подростков.

Комментарии. В предыдущем Европейском консенсусе 2014 г. [4] исследование гормонального профиля было рекомендовано через 4 ч от последнего приема левотироксина, настоящие рекомендации позволяют проводить контроль как до, так и после приема препарата. Нет убедительных доказательств в пользу одной оптимальной схемы наблюдения, основанной на исследованиях с оценкой развития нервной системы, однако проведенные исследования свидетельствуют о важности частого лабораторного мониторинга и коррекции дозы в течение первых 2-3 лет жизни. В Европейских рекомендациях предложен более частый мониторинг гормональных показателей в раннем возрасте. Лечение высокими дозами препарата (10-15 мкг/кг/сут) требует более частой коррекции дозы [23, 24].

\section{3. Лечение и мониторинг при центральном ВГ,}

- При тяжелых формах центрального ВГ (св. Т 4 менее 5 пмоль/л) для быстрой нормализации уровня св. $\mathrm{T}_{4}$ также рекомендуется начинать лечение левотироксином в максимально ранние сроки в дозах, подобных

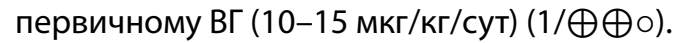

- При более легких формах центрального ВГ предлагается начинать лечение с более низкой дозы левоти-

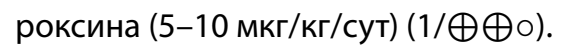

- У новорожденных с центральным ВГ рекомендуется оценивать лечение путем контроля св. $T_{4}$ и ТТГ по той же схеме, что и для первичного ВГ; уровень св. $\mathrm{T}_{4}$ должен быть выше среднего/медианного значения возрастного референсного интервала; если уровень ТТГ до начала терапии низкий, в дальнейшем можно не проводить его контроль (1/Фо०).

- При подозрении на недостаточное лечение или передозировку у пациента с центральным ВГ можно провести контроль уровня ТТГ, св. $\mathrm{T}_{3}$ или общего $\mathrm{T}_{3}$ $(1 / \bigoplus \circ \circ)$.

- Уровень св. Т У нижней границы референсного интервала, а особенно при ТТГ более 1,0 мE/л, должен учитываться при лечении.

- Когда уровень св. Т находится около или выше верхнего предела референсного интервала, следует рассмотреть вопрос о передозировке (если перед взятием крови не было приема левотироксина), особенно если имеются клинические признаки тиреотоксикоза или высокая концентрация св. $\mathrm{T}_{3}(1 / \bigoplus \circ \circ)$.

Комментарии. Данный раздел консенсуса является новым. В Российских протоколах по диагностике и лечению гипопитуитаризма у детей и подростков (2014г.) [5] рекомендуется доза левотироксина 50 мкг/м²/сут или около половины дозы, используемой при лечении первичного гипотиреоза; критерий компенсации - нормальный уровень св. $\mathrm{T}_{4^{\prime}}$ в то время как настоящий консенсус не выделяет различий в дозе препарата при первичном и центральном ВГ.

\section{4. Повторная оценка функции щЖ с целью уточнения диагноза.}

- Если в первые недели или месяцы лечения не был установлен окончательный диагноз ВГ, показана 
повторная оценка гипоталамо-гипофизарно-тиреоидной оси после 2-3 лет, особенно у детей с ЩЖ в типичном месте (a gland-in-situ) и у детей с подозрением на изолированный центральный ВГ $(1 / \bigoplus \bigoplus$ $)$.

- Для уточнения диагноза терапию левотироксином необходимо отменить постепенно в течение 4-6 нед или сразу, и через 4 нед провести повторную оценку функции ЩЖ (св. Т 4 и ТТГ).

- Если первичный гипотиреоз подтвержден только уровнем ТТГ (ТТГ больше или равен 10 мЕ/л), то следует рассмотреть возможность проведения визуализации ЩЖ и генетического исследования; если центральный ВГ вероятен (св. Т ниже нижней границы референсного интервала в сочетании с низким, нормальным или незначительно повышенным ТТГ), следует провести оценку других гормонов передней доли гипофиза и генетическое исследование.

- Если ТТГ выше верхней границы референсного интервала, но менее $10 \mathrm{mE} / л$ или св. Т находится на нижней границе референсного интервала, то необходимо продолжить наблюдение на фоне отмены препарата и провести повторное исследование еще

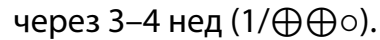

- Если ребенку с диагнозом транзиторный ВГ и ЩЖ в типичном месте требуется доза левотироксина менее 3 мкг/кг/сут в возрасте 6 мес, то повторная оценка функции может быть проведена уже в это время,

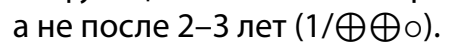

- Рекомендуется избегать использования йодсодержащих антисептиков в пери- и неонатальном периоде,

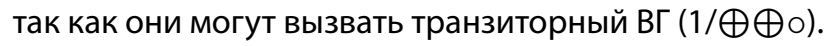

Комментарии. В последние годы неуклонно растет распространенность транзиторного ВГ. Согласно исследованиям, проведенным в 2018 г., при обследовании детей с ВГ и типичным расположением ЩЖ на долю транзиторного гипотиреоза приходится 20-54\% случаев [25, 26]. По литературным данным, к факторам, повышающим вероятность транзиторного ВГ, можно отнести пол (чаще у мальчиков) [27], низкий вес при рождении [28], неонатальную патологию, требующую интенсивной терапии [29], «легкие» формы ВГ на момент установки диагноза [30-37]. Проведенные исследования подчеркивают необходимость проведения повторного обследования для выявления детей, которые не нуждаются в длительной терапии.

Согласно Российским рекомендациям по лечению и диагностике ВГ 2014 г. [4] (и проекту клинических рекомендаций 2020 г. [5]), уточнение диагноза и решение вопроса о необходимости продолжения лечения проводится в возрасте после 1 года на фоне отмены препарата на 3-4 нед.

Новым положением Европейского консенсуса 2020 г. является ограничение применения кожных антисептиков с содержанием йода (в акушерстве и неонатологии), поскольку йод легко проникает в ЩЖ плода или новорожденного, вызывая транзиторный гипотиреоз [38, 39].

\section{5. Лечение и наблюдение беременных женщин $\mathrm{c} B \Gamma$ 。}

- У пациенток с ВГ, планирующих беременность, рекомендуется тщательно контролировать лечение левотироксином; кроме того, они должны быть проинформированы о возможности увеличения дозы

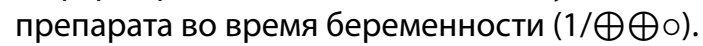

- Уровни св. Т (или общего Т 4 ) и ТТГ следует контролировать каждые 4-6 нед в течение беременности, уровень ТТГ в соответствии с современными рекомендациями по лечению гипотиреоза должен быть не выше 2,5 мЕ/л на протяжении всей беременности у пациенток, получающих лечение левотироксином $(1 / \oplus \circ \circ)$.

- У беременных женщин с центральным ВГ дозу левотироксина следует увеличивать, стремясь к уровню св. $\mathrm{T}_{4}$ выше среднего/медианного значения референсного интервала для соответствующего триместра беременности (1/Фоо).

- После родов рекомендуется снизить дозу левотироксина до прежней, которая была до беременности; дополнительно оценка функции ЩЖ должна проводиться примерно через 6 нед после родов $(1 / \bigoplus \bigoplus$ $)$.

- Bce беременные женщины должны принимать

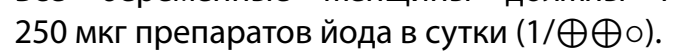

Комментарии. В Российских клинических рекомендациях по диагностике и лечению ВГ у детей [4] тактика наблюдения при беременности отсутствует. Однако в связи с возможностью беременности в подростковом возрасте данный раздел консенсуса является важным. По сравнению с Европейским консенсусом 2014 г. [2] обновленные рекомендации не выделяют целевой уровень ТТГ в зависимости от срока беременности, а рекомендуют придерживаться уровня менее 2,5 мЕ/л на всем протяжении беременности. Также к рекомендациям добавлены сведения о применении препаратов йода в дозе, рекомендованной ВОЗ.

\section{4. РЕЗУЛЬТАТЫ НЕОНАТАЛЬНОГО СКРИНИНГА И РАННЕГО НАЧАЛА ЛЕЧЕНИЯ}

\section{1. Развитие нервной системы。}

- Психомоторное развитие и успеваемость в школе должны периодически оцениваться у всех детей с ВГ; поводом для дополнительной оценки являются задержка речи, проблемы с вниманием и памятью,

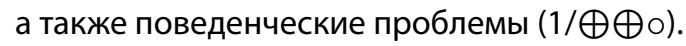

- У небольшой части детей с ВГ, у которых наблюдается значительная задержка психомоторного развития при своевременно начатом лечении, необходимо исключить другие причины интеллектуальных нарушений, кроме ВГ (1/Фоо).

- У детей с ВГ исследование слуха необходимо проводить не только в неонатальном периоде, но и позже до школьного возраста, при необходимости и во вре-

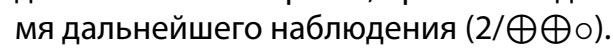

Комментарии. В настоящее время большинство детей с ВГ имеют нормальное нервно-психическое развитие и успеваемость. Однако, несмотря на своевременно начатую и адекватную терапию, у пациентов с тяжелой формой ВГ может быть незначительный когнитивный и моторный дефицит. Своевременно не диагностированное нарушение слуха может отрицательно сказаться на развитии речи, успеваемости в школе и качестве жизни. Согласно отечественным 
клиническим рекомендациям 2014 г. [4] и проекту 2020 г. [5], определены сроки диспансерного наблюдения для детей с ВГ: на первом году жизни рекомендован осмотр оториноларингологом, консультация невролога 1 раз в 3-6 мес, в 1 год - осмотр сурдологом и в возрасте 4-5 лет - логопедом, дальнейшее обследование по показаниям. Настоящий Европейский консенсус не регламентирует конкретные сроки обследования узкими специалистами.

\section{2. Развитие зоба при дисгормоногенезе ЩЖ.}

- У детей и подростков с первичным ВГ, возникшим вследствие дисгормоногенеза, могут сформироваться $30 б$ и узлы; в этих случаях целевые значения сывороточного ТТГ должны быть у нижней границы референсного интервала, для мониторинга объема ЩЖ рекомендовано периодическое

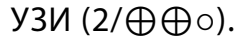

- Так как было зарегистрировано несколько случаев рака ЩЖ, в случае подозрительных узлов при УЗИ должна быть выполнена тонкоигольная аспирационная биопсия (ТАБ) (1/Фоо).

Комментарии. Данный раздел консенсуса является новым. С целью своевременного выявления узлов необходимо регулярное проведение УзИ ЩЖ (каждые 2-3 года) [3]. В Российских рекомендациях сроки выполнения УЗИ при наличии зоба определены как 1 раз в 6-12 мес $[4,5]$.

\section{3. Рост, половое созревание и фертильность.}

- Дети с несиндромальным ВГ, получающие адекватную заместительную терапию, имеют нормальный рост и половое созревание, их фертильность ничем не от-

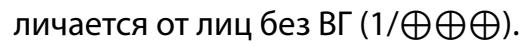

\section{4. Опорно-двигательный аппарат, обмен веществ и сердечно-сосудистая система.}

- Дети с несиндромальным ВГ, получающие адекватную заместительную терапию, также имеют нормальное состояние опорно-двигательного аппарата, обмена веществ и сердечно-сосудистой системы $(1 / \bigoplus \bigoplus$ $)$.

\section{4. Обучение пациентов и врачей, а также качество жизни, связанное со здоровьем.}

- Медицинские образовательные программы по вопросам ВГ должны совершенствоваться и регулярно

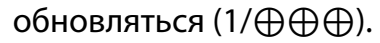

- Начиная с момента постановки диагноза необходимо обучение родителей, а в дальнейшем и самого пациента приверженности лечению; особые периоды переход во взрослое лечебное учреждение, женщины с ВГ во время беременности $(1 / \bigoplus \bigoplus \bigoplus)$.

- Приверженность лечению следует стимулировать

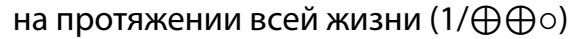

\section{5. Переход во взрослое лечебное учреждение.}

- При переводе пациентов из педиатрического лечебного учреждения в терапевтическое основными целями являются непрерывность лечения, качество жизни, улучшение понимания проблемы ВГ $(1 / \bigoplus \bigoplus \bigoplus)$.
Комментарии. Следует уделять должное внимание переводу пациента из педиатрической службы во взрослое лечебное учреждение, поскольку он может быть связан со снижением приверженности к лечению и нерегулярным последующим наблюдением, что может привести к ухудшению течения заболевания.

\section{5. ГЕНЕТИКА ВРОЖДЕННОГО ГИПОТИРЕОЗА, ГЕНЕТИЧЕСКОЕ КОНСУЛЬТИРОВАНИЕ И ДОРОДОВОЕ ВЕДЕНИЕ}

\section{1. Критерии генетического консультирования,}

- Генетическое консультирование должно быть персонализированным для каждого пациента с ВГ и прово-

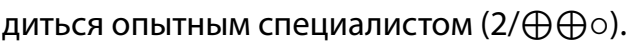

- Консультирование должно включать в себя обсуждение характера наследования заболевания и риска возникновения повторных случаев ВГ в семье. Если известен семейный анамнез, то установления генети-

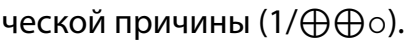

- Семьи, имеющие ребенка с ВГ, должны быть информированы о двух самых частых формах первичного ВГ - дисгенезии ЩЖ и дисгормоногенезе, при включении определения св. Т В неонатальный скри-

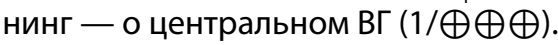

\section{2. Генетика ВГ.}

- Если проводится генетическое исследование, то его целью должно быть улучшение диагностики, лечения

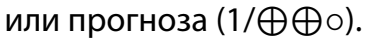

- До начала исследования следует обсудить с родителями/семьей возможности и ограничения генетиче-

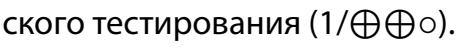

- По возможности генетическое исследование должно проводиться с помощью новых методов, таких как сравнительная геномная гибридизация (CGH), секвенирование нового поколения панели генов (NGS) или

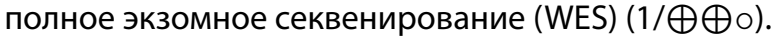

- Генетическому исследованию должно предшествовать тщательное фенотипическое описание пациента с ВГ,

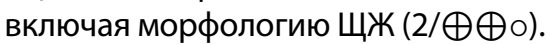

- Не только пациенты с дисгормоногенезом, но и семейные случаи дисгенезии ЩЖ и центрального ВГ должны подлежать генетическому обследованию $(1 / \bigoplus \bigoplus$ $)$

- Сочетание ВГ с синдромальной патологией требует молекулярно-генетической верификации для выяв-

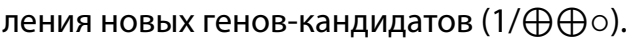

- Необходимо проведение дальнейших исследований для определения категорий пациентов, которым потребуются новые диагностические возможности $(2 / \bigoplus \bigoplus \circ)$.

Комментарии. Определить генетическую основу заболевания позволяет молекулярно-генетическое обследование. В представленных Европейских рекомендациях вопросу генетики ВГ уделяется большее внимание. Перед направлением пациента на генетическое исследование необходимо обратить внимание на наличие фенотипических особенностей. Поиск мутаций генов-кандидатов, ответственных за развитие врожденного гипотиреоза, целесообразно проводить во всех семейных случаях ВГ. 


\section{3. Антенатальная диагностика, оценка функции ЩЖ плода и лечение гипотиреоза плода.}

- Антенатальная диагностика рекомендуется в случаях зоба, выявленного при УЗИ плода $(1 / \bigoplus \bigoplus \bigoplus)$; семейного случая ВГ, обусловленного дисгормоногенезом

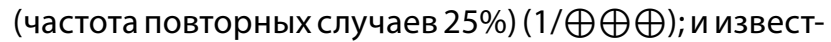
ных дефектах генов, участвующих в развитии щЖ

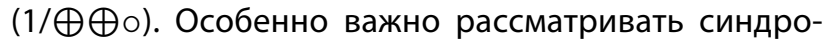
мальные случаи с потенциальной смертностью и эмбриональным мозаицизмом (например, мутации/делеции гена NKX2-1 и тяжелая легочная дисфункция). Семейные случаи ВГ, обусловленные дисгенезией (2\% семейных случаев), требуют дальнейшего изучения для определения целесообразности и клинической значимости антенатального выявления.

- Для оценки объема ЩЖ плода рекомендуется проведение УЗИ в сроке от 20 до 22 нед беременности этим методом могут быть выявлены 306 или дисгенезия, в том числе отсутствие ткани ЩЖ. При оценке размеров ЩЖ следует учитывать гестационный возраст, в случаях зоба должен быть измерен объем щЖ $(1 / \bigoplus \bigoplus \bigoplus)$.

- Если у плода диагностирован 3об, пренатальная помощь должна оказываться в специализированном перинатальном центре $(1 / \bigoplus \bigoplus \bigoplus)$.

- В качестве эталонного метода оценки функции ЩЖ плода рекомендуется кордоцентез, а не амниоцентез. Данное обследование следует проводить только в том случае, если рассматривается вопрос о пренатальном вмешательстве $(1 / \bigoplus \bigoplus \bigoplus)$.

В большинстве случаев состояние ЩЖ плода можно оценить на основании УЗ-критериев, поэтому забор крови плода требуется только в исключительных случаях $(2 / \oplus \bigoplus 0)$.

- У эутиреоидной беременной женщины с прогрессирующим многоводием, при больших размерах зоба у плода и/или с окклюзией трахеи плода, а также риском преждевременных родов, рекомендуется лечение плода внутриамниотическими инъекциями тироксина; у беременной женщины с гипотиреозом рекомендуется лечить женщину (а не плод) левотироксином $(1 / \bigoplus \bigoplus$ ).
- При неиммунном гипотиреозе и наличии зоба у плода на фоне многоводия рекомендуются внутриамниотические инъекции левотироксина для уменьшения размеров ЩЖ плода. Инъекции должны выполняться многопрофильными бригадами специалистов $(1 / \bigoplus \bigoplus \bigoplus)$. Экспертная группа предлагает использовать 10 мкг/кг расчетной массы плода в течение 15 дней в виде внутриамниотического введения препарата. Риск для плода и психологическая нагрузка на родителей должны быть учтены при оценке риска/ пользы (2/®оо).

Комментарии. В отличие от Европейских рекомендаций 2014 г., это во многом новый раздел, в частности, определены дозы левотироксина для Внутриутробного лечения зоба плода. В Российской Федерации данный метод лечения не используется (нет зарегистрированных парентеральных форм левотироксина).

\section{ЗАКЛЮЧЕНИЕ}

Представленные материалы отражают современные взгляды на диагностику, лечение и наблюдение за пациентами с ВГ. Расширены разделы, посвященные молекулярно-генетическому обследованию пациентов/семей и наблюдению за беременными пациентками с ВГ, а также введены новые разделы, в частности, наблюдение за детьми с ВГ и зобом и центральным гипотиреозом.

\section{ДОПОЛНИТЕЛЬНАЯ ИНФОРМАЦИЯ}

Источники финансирования. Работа выполнена по инициативе авторов без привлечения финансирования.

Конфликт интересов. Авторы декларируют отсутствие явных и потенциальных конфликтов интересов, связанных с содержанием настоящей статьи.

Участие авторов. Все авторы одобрили финальную версию статьи перед публикацией, выразили согласие нести ответственность за все аспекты работы, подразумевающую надлежащее изучение и решение вопросов, связанных с точностью или добросовестностью любой части работы.

\section{СПИСОК ЛИТЕРАТУРЫ | REFERENCES}

1. Dussault JH, Coulombe P, Laberge C, et al. Preliminary report on a mass screening program for neonatal hypothyroidism. J Pediatr. 1975;86(5):670-674 doi: https://doi.org/10.1016/s0022-3476(75)80349-0

2. Léger J, Olivieri A, Donaldson M, et al. European Society for Paediatric Endocrinology Consensus Guidelines on Screening, Diagnosis, and Management of Congenital Hypothyroidism. Horm Res Paediatr. 2014;81(2):80-103. doi: https://doi.org/10.1159/000358198

3. van Trotsenburg P, Stoupa A, Léger J, et al. Congenital Hypothyroidism: A 2020 - 2021 Consensus Guidelines UpdateAn ENDO-European Reference Network Initiative Endorsed by the European Society for Pediatric Endocrinology and the European Society for Endocrinology. Thyroid. 2021;31(3):387-419. doi: https://doi.org/10.1089/thy.2020.0333

4. Дедов И.И., Петеркова В.А. Федеральные клинические рекомендации (протоколы) по ведению детей с эндокринными заболеваниями. - M.; 2014. - C. 183-214. [Dedov II, Peterkova VA.
Federal'nye klinicheskie rekomendacii (protokoly) po vedeniju detej s jendokrinnymi zabolevanijami. Moscow; 2014. P. 183-214. (In Russ.)].

5. Проект клинических рекомендачий «Диагностика и лечение врожденного гипотиреоза». Разработчик клинических рекомендаций: Общественная организация «Российская ассоциация эндокринологов». 2020. [Proekt klinicheskih rekomendacij «Diagnostika i lechenie vrozhdennogo gipotireoza». Razrabotchik klinicheskih rekomendacij: Obshhestvennaja organizacija «Rossijskaja associacija jendokrinologov». 2020. (In Russ.)]. Доступно по: https://rae-org.ru/system/files/documents/ pdf/klinicheski_rekomendacii_vg_ot_03.06.2020.pdf

6. Swiglo BA, Murad MH, Schünemann HJ, et al. A Case for Clarity, Consistency, and Helpfulness: State-of-the-Art Clinical Practice Guidelines in Endocrinology Using the Grading of Recommendations, Assessment, Development, and Evaluation System. J Clin Endocrinol Metab. 2008;93(3):666-673. doi: https://doi.org/10.1210/jc.2007-1907 
7. Дедов И.И., Безлепкина О.Б., Вадина Т.А., и др. Скрининг на врожденный гипотиреоз в Российской Федерации // Проблемы эндокринологии. - 2018. - Т. 64. - №1. C. 14-20. [Dedov II, Bezlepkina OB, Vadina TA, et al. Screening for congenital hypothyroidism in the Russian Federation. Problems of Endocrinology. 2018;64(1):14-20. (In Russ.)]. doi: https://doi.org/10.14341/probl8752

8. McGrath N, Hawkes CP, Mayne P, Murphy NP. Optimal Timing of Repeat Newborn Screening for Congenital Hypothyroidism in Preterm Infants to Detect Delayed ThyroidStimulating Hormone Elevation. J Pediatr. 2019;205(2):77-82. doi: https://doi.org/10.1016/j.jpeds.2018.09.044

9. LaFranchi SH. Screening Preterm Infants for Congenital Hypothyroidism: Better the Second Time Around. J Pediatr. 2014;164(6):1259-1261. doi: https://doi.org/10.1016/j.jpeds.2014.02.031

10. Fort $\mathrm{P}$, Lifshitz F, Bellisario $\mathrm{R}$, et al. Abnormalities of thyroid function in infants with Down syndrome. J Pediatr. 1984;104:545-549. doi: https://doi.org/10.1016/s0022-3476(84)80544-2

11. Purdy $I B$, Singh $N$, Brown $W L$, et al. Revisiting early hypothyroidism screening in infants with Down syndrome. J Perinatol. 2014;34:936-940. doi: https://doi.org/10.1038/jp.2014.116

12. Olivieri A, Medda E, De Angelis S, et al. High Risk of Congenital Hypothyroidism in Multiple Pregnancies. J Clin Endocrinol Metab. 2007;92(8):3141-3147. doi: https://doi.org/10.1210/jc.2007-0238

13. Perry R, Heinrichs C, Bourdoux P, et al. Discordance of Monozygotic Twins for Thyroid Dysgenesis: Implications for Screening and for Molecular Pathophysiology. J Clin Endocrinol Metab. 2002;87(9):4072-4077. doi: https://doi.org/10.1210/jc.2001-011995

14. Medda E, Vigone MC, Cassio A, et al. Neonatal Screening for Congenital Hypothyroidism: What Can We Learn From Discordant Twins? J Clin Endocrinol Metab. 2019;104(12):5765-5779. doi: https://doi.org/10.1210/jc.2019-00900

15. Приказ Минздравсоцразвития России №185 «О массовом обследовании новорожденных детей на наследственные заболевания» от 22.03.2006 г. [Prikaz Minzdravsocrazvitija Rossii №185 «O massovom obsledovanii novorozhdennyh detej na nasledstvennye zabolevanija». 22 March 2006. (In Russ.)].

16. Trumpff C, De Schepper J, Vanderfaeillie J, et al. Neonatal thyroid-stimulating hormone concentration and psychomotor development at preschool age. Arch Dis Child. 2016;101(12):1100-1106. doi: https://doi.org/10.1136/archdischild-2015-310006

17. Lain SJ, Bentley JP, Wiley V, et al. Association between borderline neonatal thyroid-stimulating hormone concentrations and educational and developmental outcomes: a population-based record-linkage study. Lancet Diabetes Endocrinol. 2016;4(9):756-765. doi: https://doi.org/10.1016/S2213-8587(16)30122-X

18. Vigone MC, Capalbo D, Weber G, Salerno M. Mild Hypothyroidism in Childhood: Who, When, and How Should Be Treated? J Endocr Soc. 2018;2(9):1024-1039. doi: https://doi.org/10.1210/js.2017-00471

19. De Silva A, Jong I, McLean G, et al. The role of scintigraphy and ultrasound in the imaging of neonatal hypothyroidism: 5 -year retrospective review of single-centre experience. $J$ Med Imaging Radiat Oncol. 2014;58(4):422-430. doi: https://doi.org/10.1111/1754-9485.12166

20. Keller-Petrot I, Leger J, Sergent-Alaoui A, de LabriolleVaylet C. Congenital Hypothyroidism: Role of Nuclear Medicine. Semin Nucl Med. 2017;47(2):135-142. doi: https://doi.org/10.1053/j.semnuclmed.2016.10.005

21. Albert BB, Heather N, Derraik JGB, et al. Neurodevelopmental and Body Composition Outcomes in Children With Congenital Hypothyroidism Treated With High-Dose Initial Replacement and Close Monitoring. J Clin Endocrinol Metab. 2013;98(9):3663-3670. doi: https://doi.org/10.1210/jc.2013-1903

22. Aleksander PE, Brückner-Spieler M, Stoehr A-M, et al. Mean High-Dose I-Thyroxine Treatment Is Efficient and Safe to Achieve a Normal IQ in Young Adult Patients With Congenital Hypothyroidism. J Clin Endocrinol Metab. 2018;103(4):1459-1469. doi: https://doi.org/10.1210/jc.2017-01937

23. Tuhan $\mathrm{H}$, Abaci A, Cicek $\mathrm{G}$, et al. Levothyroxine replacement in primary congenital hypothyroidism: the higher the initial dose the higher the rate of overtreatment. $J$ Pediatr Endocrinol Metab. 2016;29(2):133-138. doi: https://doi.org/10.1515/jpem-2015-0047.

24. Craven M, Frank GR. Does initial dosing of levothyroxine in infants with congenital hypothyroidism lead to frequent dose adjustments secondary to iatrogenic hyperthyroidism on follow-up? J Pediatr Endocrinol Metab. 2018;31(6):597-600. doi: https://doi.org/10.1515/jpem-2017-0513

25. Saba C, Guilmin-Crepon S, Zénaty D, et al. Early Determinants of Thyroid Function Outcomes in Children with Congenital Hypothyroidism and a Normally Located Thyroid Gland: A Regional Cohort Study. Thyroid. 2018;28(8):959-967. doi: https://doi.org/10.1089/thy.2018.0154

26. Oron T, Lazar L, Ben-Yishai S, et al. Permanent vs Transient Congenital Hypothyroidism: Assessment of Predictive Variables. J Clin Endocrinol Metab. 2018;103(12):4428-4436. doi: https://doi.org/10.1210/jc.2018-00362

27. Oren A, Wang MK, Brnjac L, et al. Mild neonatal hyperthyrotrophinaemia: 10-year experience suggests the condition is increasingly common but often transient. Clin Endocrinol (Oxf). 2013;79(6):832-837. doi: https://doi.org/10.1111/cen.12228

28. Jung JM, Jin HY, Chung ML. Feasibility of an Early Discontinuation of Thyroid Hormone Treatment in Very-Low-Birth-Weight Infants at Risk for Transient or Permanent Congenital Hypothyroidism. Horm Res Paediatr. 2016;85(2):131-139. doi: https://doi.org/10.1159/000443399

29. Korzeniewski SJ, Grigorescu V, Kleyn M, et al. Transient Hypothyroidism at 3-Year Follow-Up among Cases of Congenital Hypothyroidism Detected by Newborn Screening. J Pediatr. 2013;162(1):177-182. doi: https://doi.org/10.1016/j.jpeds.2012.06.050

30. Langham S, Hindmarsh P, Krywawych S, Peters C. Screening for congenital hypothyroidism: comparison of borderline screening cut-off points and the effect on the number of children treated with levothyroxine. Eur Thyroid J. 2013;2:180-186. doi: https://doi.org/10.1159/000350039

31. Kara C, Günindi F, Can Yılmaz G, Aydın M. Transient Congenital Hypothyroidism in Turkey: An Analysis on Frequency and Natural Course. J Clin Res Pediatr Endocrinol. 2016;8(2):170-179. doi: https://doi.org/10.4274/jcrpe.2345

32. Ford GA, Denniston S, Sesser D, Skeels MR, LaFranchi SH. Transient versus Permanent Congenital Hypothyroidism after the Age of 3 Years in Infants Detected on the First versus Second Newborn Screening Test in Oregon, USA. Horm Res Paediatr. 2016;86(3):169-177. doi: https://doi.org/10.1159/000448658

33. Kang MJ, Chung HR, Oh YJ, et al. Three-year follow-up of children with abnormal newborn screening results for congenital hypothyroidism. Pediatr Neonatol. 2017;58(5):442-448. doi: https://doi.org/10.1016/j.pedneo.2017.01.002

34. Park IS, Yoon JS, So CH, et al. Predictors of transient congenital hypothyroidism in children with eutopic thyroid gland. Ann Pediatr Endocrinol Metab. 2017;22(2):115-118. doi: https://doi.org/10.6065/apem.2017.22.2.115

35. Zdraveska N, Zdravkovska M, Anastasovska V, et al. Diagnostic reevaluation of congenital hypothyroidism in Macedonia: predictors for transient or permanent hypothyroidism. Endocr Connect. 2018;7(2):278-285. doi: https://doi.org/10.1530/EC-17-0332

36. Fu C, Luo S, Li Y, et al. The incidence of congenital hypothyroidism $(\mathrm{CH})$ in Guangxi, China and the predictors of permanent and transient CH. Endocr Connect. 2017;6(8):926-934. doi: https://doi.org/10.1530/EC-17-0289

37. Razavi Z, Mohammadi L. Permanent and Transient Congenital Hypothyroidism in Hamadan West Province of Iran. Int J Endocrinol Metab. 2017;14(4):e38256. doi: https://doi.org/10.5812/ijem.38256

38. Fernández Ruiz A, García-Guereta L, Benito Bartolomé $F$, et al. Alteraciones de la función tiroidea en niños con cardiopatía congénita tras la realización de cateterismo con contrastes yodados. Rev Española Cardiol. 2000;53(4):517-524. doi: https://doi.org/10.1016/S0300-8932(00)75122-0

39. Thaker VV, Galler MF, Marshall AC, et al. Hypothyroidism in Infants With Congenital Heart Disease Exposed to Excess lodine. J Endocr Soc. 2017;1(8):1067-1078. doi: https:/doi.org/10.1210/js.2017-00174 


\section{ИНФОРМАЦИЯ ОБ АВТОРАХ [AUTHORS INFO]}

*Шрёдер Екатерина Владимировна [Ekaterina V. Shreder, MD]; клинический аспирант, врач детский эндокринолог ФГБУ «НМИЦ эндокринологии» МЗ РФ; адрес: Россия; 117036, Москва, ул. Дм. Ульянова, д. 11 [address: 11 Dm. Ulyanova street, 117036 Moscow, Russia] ORCID: http://orcid.org/0000-0003-0031-1389; eLibrary SPIN: 7997-2501; e-mail: evshreder@bk.ru

Ширяева Татьяна Юрьевна, К.м.н. [Tatiana Y. Shiryaeva, MD, PhD]; ORCID: https://orcid.org/0000-0002-2604-1703; eLibrary SPIN: 1322-0042

Нагаева Елена Витальевна, к.M.н. [Elena V. Nagaeva, MD, PhD]; ORCID: https://orcid.org/0000-0001-6429-7198; eLibrary SPIN: 4878-7810

Безлепкина Ольга Борисовна, д.М.н., профессор [Olga B. Bezlepkina, MD, PhD, Professor];

ORCID: https://orcid.org/0000-0001-9621-5732; eLibrary SPIN: 3884-0945

*Автор, ответственный за переписку.

\section{ЦИТИРОВАТЬ}

Шрёдер Е.В., Ширяева Т.Ю., Нагаева Е.В., Безлепкина О.Б. Клинические рекомендации по врожденному гипотиреозу Европейского общества детских эндокринологов (ESPE) и Европейского эндокринологического общества (ESO): основные положения и комментарии // Клиническая и экспериментальная тиреоидология. — 2021. — Т. 17. — №2. — C. 4-12. doi: https://doi.org/10.14341/ket12703

Рукопись получена:13.04.2021

Одобрена к публикации: 06.07.2021

\section{TO CITE THIS ARTICLE}

Shreder EV, Shiryaeva TY, Nagaeva EV, Bezlepkina OB. Consensus guidelines of congenital hypothyroidism by the European Society for pediatric endocrinology and the European Society for Endocrinology: key points and comments. Clinical and experimental thyroidology. 2021;17(2):4-12. doi: https://doi.org/10.14341/ket12703

Received: 13.04.2021. Accepted: 06.07.2021 